\title{
In Situ Synchrotron-Based IR Microspectroscopy To Study Catalytic Reactions in Zeolite Crystals**
}

\author{
Eli Stavitski, Marianne H. F. Kox, Ingmar Swart, Frank M. F. de Groot, and \\ Bert M. Weckhuysen*
}

In recent years a number of in situ microspectroscopic techniques have been explored to investigate catalytic reactions taking place in heterogeneous catalysts in a timeand space-resolved manner. ${ }^{[1-8]}$ These spectroscopic methods have proven to be very successful in elucidating valuable structure-function relationships for acid-base catalytic reactions. In the work of Roeffaers et al. fluorescence microscopy has been applied to demonstrate the crystal-face-dependent catalysis on layered double hydroxide (LDH) materials. ${ }^{[1]}$ By this elegant approach one can track interconversion processes of individual molecules in catalytic solids and obtain indirect data on the chemical nature of the reaction products formed, provided they display fluorescence. For example, the red shift of the emission bands of specific carbocations formed was used to visualize the degree of oligomerization of cyclic alcohols in individual H-ZSM-5 zeolite particles. ${ }^{[3]}$

Alternatively, one can use in situ optical microspectroscopy to monitor the course of chemical reactions within individual zeolite particles. By using this approach, which features a spatial resolution on par with that of the fluorescence method, it is possible to map the reaction products of styrene oligomerization within the micropores of H-ZSM-5 zeolite crystals at different positions in the catalyst material. In addition, polarization-dependent experiments provide insight in the molecular orientation of the reaction products formed, and by altering the molecular structure of the styrene compounds different reactivity trends can be observed. Furthermore, the acquired in situ optical absorption spectra make it possible to differentiate between the dimeric and trimeric styrene oligomers formed.

However, extra measures are required if one wants to unambiguously unravel the chemical nature of the species

[*] Dr. E. Stavitski, M. H. F. Kox, I. Swart, Dr. F. M. F. de Groot, Prof. Dr. ir. B. M. Weckhuysen

Inorganic Chemistry and Catalysis Group

Department of Chemistry, Utrecht University

Sorbonnelaan 16, 3584 CA Utrecht (The Netherlands)

Fax: (+31) 30-251-1027

E-mail: b.m.weckhuysen@uu.nl

[**] We thank the Dutch National Science Foundation (NWO-CW VICI and TOP grants) and the Netherlands Research School Combination on Catalysis (NRSC-C) for financial support. The NSLS is supported by the U.S. Department of Energy, Office of Science, Office of Basic Energy Sciences, under contract DE-AC0298CH10886. Randy Smith and Dr. Lisa Miller are acknowledged for their assistance with the IR measurements. We also thank Dr. Tom Visser for fruitful discussions.

9 Supporting information for this article is available on the WWW under http://www.angewandte.org or from the author. formed during reaction. This is especially true when comparison with suitable reference compounds fails or when reference compounds are difficult to synthesize, as is the case where reaction products and/or reaction intermediates are ions or radicals. The microspectroscopic methodology described above therefore will certainly benefit from a complimentary technique capable of providing direct insight into the chemical nature of the reagent molecules, potential reaction intermediates, and reaction products. One of the plausible powerful candidates to fill this gap is infrared (IR) microscopy, which is based on the coupling of an IR spectrometer and an optical microscope. The latter ensures that only a localized area of the catalyst sample is probed. This technique, first applied to map the boron distribution in B-ZSM-5 ${ }^{[9]}$ has been established as a valuable tool in zeolite studies. Examples of its use in the field of microporous materials include studies of the decomposition of the organic template ${ }^{[10]}$ diffusion, ${ }^{[11-13]}$ and mass transport. ${ }^{[14]}$ However, to the best of our knowledge, IR microscopy has never been applied to the study of catalytic reactions in situ within zeolite grains or crystals in a space- and time-resolved manner.

Here we report on the first in situ investigation of catalytic reactions in zeolite crystals by IR microspectroscopy. Styrene oligomerization, chosen as a probe reaction, has been thoroughly studied by means of UV/Vis and confocal fluorescence microspectroscopy. ${ }^{[4,5]}$ To boost the low spatial resolution (about $20 \mu \mathrm{m}$ ), which is likely to be the most prohibiting limitation of the conventional IR microscope, we used synchrotron light with a brightness 100-1000 times higher than that of a thermal (globar) source. ${ }^{[15]}$ Owing to the improved characteristics of a synchrotron-based setup, the resolution could be brought to diffraction-limited values, ${ }^{[16]}$ making it comparable to that of UV/Vis microspectroscopy. ${ }^{[4]}$ The optical window of the in situ spectroscopic cell used previously for optical ${ }^{[4]}$ and fluorescence microscopic studies ${ }^{[5]}$ was replaced by an IR-transparent material, with no extra modifications, ensuring full comparability of the results with those obtained with the two other microspectroscopic techniques (Figure 1).

In a first set of experiments coffin-shaped H-ZSM-5 crystals $(100 \mu \mathrm{m} \times 20 \mu \mathrm{m} \times 20 \mu \mathrm{m})$ were exposed to 4-fluorostyrene and heated to $373 \mathrm{~K}$ for $10 \mathrm{~min}$, and the IR spectrum from the $5 \mu \mathrm{m} \times 5 \mu \mathrm{m}$ region in the center of the crystal was recorded (Figure 2a). Two sharp features, namely at $1534 \mathrm{~cm}^{-1}$ (with the shoulder at $1521 \mathrm{~cm}^{-1}$ ) and at $1510 \mathrm{~cm}^{-1}$ are visible in the spectrum. The broad bands $\left(1600-1700 \mathrm{~cm}^{-1}\right.$ and $1000-1300 \mathrm{~cm}^{-1}$ ) can be attributed to the harmonics of the zeolite framework. ${ }^{[17]}$ By comparing this IR spectrum with that of 4-fluorostyrene (Figure $2 \mathrm{~b}$ ) one can see that the band 


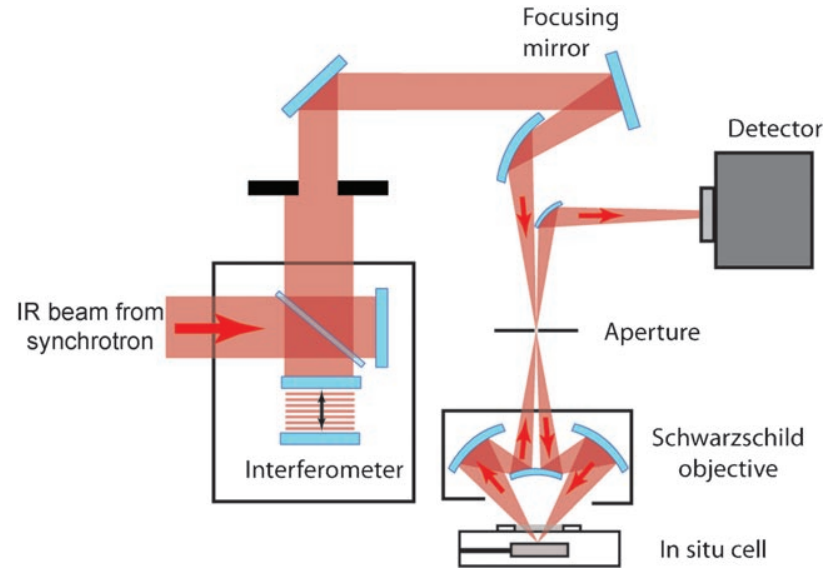

Figure 1. Construction of a scanning IR microspectrometer system in reflection mode. The microscope uses a Schwarzschild-type objective. An area of the sample defined by the aperture is spectroscopically sampled by the instrument, and an image is built-up by rasterscanning the specimen through the focused beam.

at $1510 \mathrm{~cm}^{-1}$ coincides with that of the reactant. When the spectra are recorded in situ during the reaction (Figure $2 \mathrm{c}$ ) in the in situ cell at $373 \mathrm{~K}$, the intensity of the latter band decreases, while the IR band at around $1534 \mathrm{~cm}^{-1}$ increases. Similar results were obtained when nonsubstituted styrene or 4-chlorostyrene were used (see the Supporting Information).

It was shown previously that when H-ZSM-5 zeolite is exposed to styrene compounds at 353-423 K, oligomerization takes place. ${ }^{[18-20]}$ This process is accompanied by an intense coloring of the zeolite material, ${ }^{[19]}$ owing to the formation of light-absorbing carbocationic species, as illustrated in Figure $3 \mathrm{a}$. According to the proposed reaction scheme, ${ }^{[21,22]}$ the protonation of styrene on zeolite Brønsted acid sites is followed by the attachment of another styrene molecule to form a dimeric carbocation. The reaction can proceed further towards trimeric species, with the extent determined by the pore geometry of the zeolite ${ }^{[4]}$ and the molecular characteristics of the styrene derivative. ${ }^{[5]}$ Within this reaction scheme, it is reasonable to assume that the $1534 \mathrm{~cm}^{-1}$ band arising in the course of the reaction is due to the styrene oligomeric species.

Optical microspectroscopy experiments carried out using polarized light demonstrated a strong polarization dependence of the optical absorption bands. ${ }^{[4]}$ This experiment can now be reproduced making use of the IR microscopic setup since synchrotron radiation intrinsically retains a high degree of polarization. Figure $3 \mathrm{~b}$ shows the in situ IR spectra of the H-ZSM-5 crystal loaded with fluorostyrene, which are taken with the light polarization parallel and perpendicular to the long crystal axis. Based on these measurements one can see that the intensity of the $1534 \mathrm{~cm}^{-1}$ band depends dramatically upon IR light polarization, while the $1510 \mathrm{~cm}^{-1}$ band remains unchanged. One could expect such polarization dependence from an elongated molecule aligned with the zeolite channels, confirming the spectroscopic assignment based on the UV/Vis microspectroscopic measurements. The less significant intensity changes of the band at $1510 \mathrm{~cm}^{-1}$ can be rationalized by

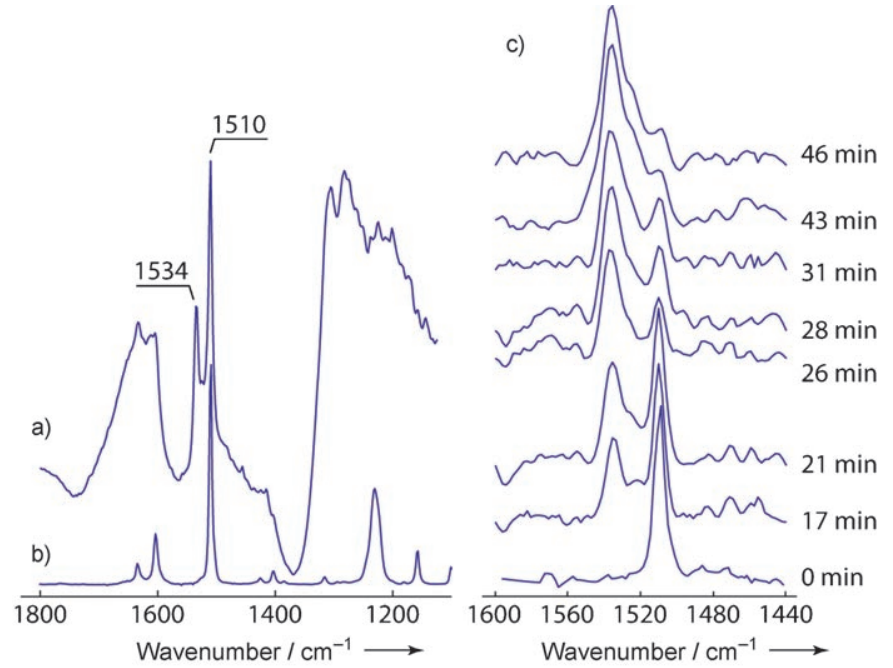

Figure 2. a) IR spectrum of an individual H-ZSM-5 crystal in contact with 4-fluorostyrene. b) IR spectrum of liquid 4-fluorostyrene. c) 1440 $1600 \mathrm{~cm}^{-1}$ region of the IR spectra taken in situ during the oligomerization reaction of 4-fluorostyrene. The spectra are corrected for background signals. a)

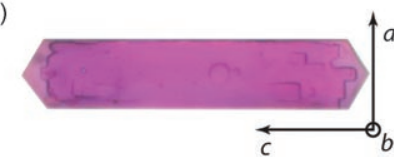

b)

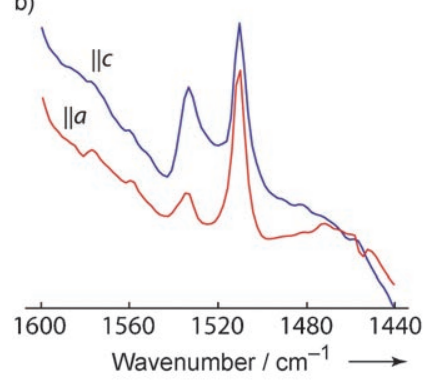

c)

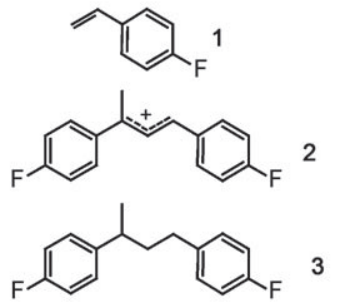

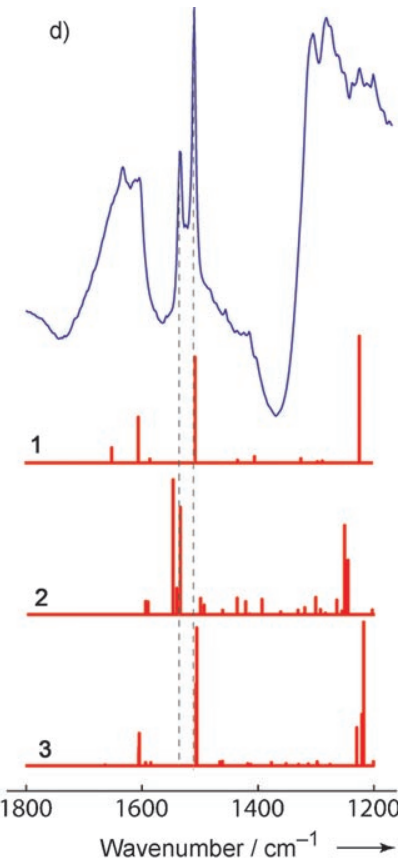

Figure 3. a) Optical microphotograph of the H-ZSM-5 crystal after reaction with 4-fluorostyrene. b) In situ IR spectra recorded with two different polarizations as indicated in (a). c) Chemical structures of 4-fluorostyrene monomer $\mathbf{1}$ and possible dimeric reaction products 2 and 3. d) Experimental and calculated spectra for structures 1-3 as shown in (c).

smaller molecular dimensions of the styrene monomer, which allow for less strict molecular alignment in the pores.

As mentioned above, in situ UV/Vis microspectroscopy provides evidence for the formation of carbocationic oligomers during the oligomerization reaction. The reaction pro- 
ducts can be identified based on a comparison with reference carbocations, obtained, for example, in liquid acidic media. However, as the isolation of the charged species is not straightforward, it can be difficult to determine the chemical structure. In the present case, the coloration arising upon zeolite-styrene interaction was attributed to the formation of 1,3-bisphenyl-2-buten-1-ylium cation (2). The neutral dimerization product shows optical absorption in the UV region and is difficult to differentiate from the reagents. To verify the assignment, IR spectra of 4-methoxystyrene and related compounds absorbed on zeolites were compared with those calculated with ab initio methods. ${ }^{[17]}$ However, while a good agreement was obtained in the case of a reference system (1,3-diphenylpropene), one notices conspicuous discrepancies between theoretical and experimental spectra in the case of 4methoxystyrene presented therein. ${ }^{[17]}$

To unravel the origin of the IR band at $1534 \mathrm{~cm}^{-1}$, geometry optimizations and vibrational frequency calculations were performed within the framework of density functional theory (DFT) for the reagent and possible products. Excellent agreement between the calculated and experimental spectrum was obtained for liquid 4-fluorostyrene (1) (see the Supporting Information). The most intense band at $1510 \mathrm{~cm}^{-1}$ coincides with the band observed during the styrene oligomerization reaction (Figure $3 \mathrm{~d}$ ). Subsequently the vibrational spectrum of 1,3-bis(4-fluorophenyl)2-buten-1-ylium cation was calculated. From the comparison with the experimental spectra, one can see two bands $\left(1549 \mathrm{~cm}^{-1}\right.$ and $\left.1538 \mathrm{~cm}^{-1}\right)$ in the calculated spectrum, very close to the band observed experimentally at $1534 \mathrm{~cm}^{-1}$. This finding as well as the spectral changes during the reaction (Figure 2c) corroborates the assignment to the conjugated carbocationic species 2 (Figure $3 \mathrm{c}$ ). The second band of the doublet in the calculated spectrum is likely to give rise to the shoulder observed in the time-resolved spectra at $1521 \mathrm{~cm}^{-1}$ (Figure 2). In addition, as the primary product of the styrene oligomerization is 1,3-bis(4-fluorodiphenyl)butane $\mathbf{3}$ (Figure $3 \mathrm{c}),{ }^{[18]}$ the IR spectrum was calculated for this compound. From inspection of the resulting spectrum (Figure $3 \mathrm{~d}$ ) one can see that the position of the most intense band matches that of 4-fluorostyrene. It implies that had 1,3-bis(4-fluorodiphenyl)butane been the only reaction product formed, it would be undistinguishable in the catalytic experiments with IR microscopy since the other IR bands are obscured by the vibrations of the zeolite framework. Nonetheless, its formation cannot be ruled out completely based on our data. Moreover, calculations performed for other possible carbocationic reaction intermediates ${ }^{[20]}$ suggest that the 1,3-bis(4fluorophenyl)-2-buten-1-ylium cation is the main reaction product (see the Supporting Information).

As mentioned before, the band at $1534 \mathrm{~cm}^{-1}$ is strongly polarized. A commonly accepted intergrowth structure of the ZSM-5 crystals implies three subunits rotated by $90^{\circ}$ around their common [001] crystallographic axis. With this model, the crystal face that was probed contains straight and zigzag channels, channels aligned parallel to the $a$ and $b$ direction, respectively (Figure $3 \mathrm{a}$ ) ${ }^{[23-25]}$ Based on the light-polarization dependence of the optical absorption, only straight channels of ZSM-5 were found to host the oligomerization products. ${ }^{[4]}$
In the present case, the intensity of the IR band associated with the dimer is higher with the light polarized along the $c$ direction, implying that the transition moment associated with the vibrational mode is likely to be directed perpendicular to the long molecular axis of the dimeric carbocation. It is interesting to notice that the electronic dipole moment associated with optical transitions coincides with the long molecular dimension, ${ }^{[4]}$ i.e., orthogonal to the vibrational one.

In a second series of experiments, the distribution of the reaction product, that is, 1,3-bis(4-fluorophenyl)-2-buten-1ylium cation, over the H-ZSM-5 crystal was mapped by scanning the individual crystal under the IR microscope. The spatial map of the $1534 \mathrm{~cm}^{-1}$ band is shown in Figure $4 \mathrm{~b}$. UV/

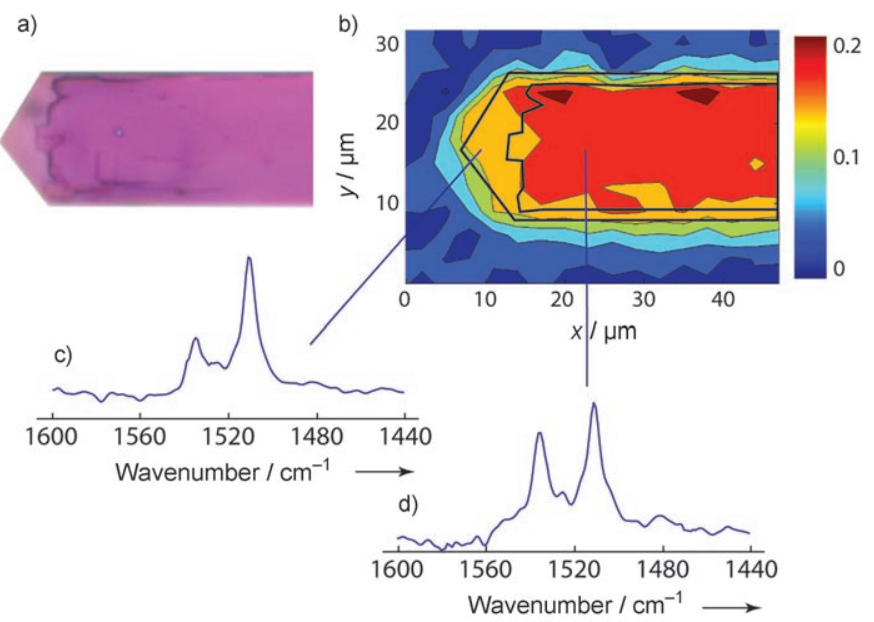

Figure 4. a) A fragment of optical microphotograph of the ZSM-5 crystal after reaction with 4-fluorostyrene. b) Intensity of the IR band at $1534 \mathrm{~cm}^{-1}$ mapped over the crystal after reaction. c,d) IR spectra taken from the edge and the body of the crystal, demonstrating differences in the intensity ratio of the bands.

Vis microspectroscopy results have suggested that dimeric carbocations are exclusively formed in the case of 4-fluorostyrene, ${ }^{[5]}$ with lower concentrations at the edges of the crystals compared to that in the center. The intergrowth structure of ZSM- ${ }^{[23-25]}$ entails that the two-dimensional channel network is oriented nonuniformly, i.e., at the edges of the crystal straight pores, open to the top surface of the crystal are accessible. On the contrary, in the center region of the crystals zigzag pores are accessible. A nonuniform product distribution was rationalized in terms of blockage of straight versus zigzag pores, as discussed in previous publications. ${ }^{[4,5]}$ The spatially resolved IR spectra shown in Figure $4 \mathrm{c}, \mathrm{d}$ are fully in line with the optical absorption results, with lower intensity of the $1534 \mathrm{~cm}^{-1}$ band at the edges as compared to the body of the crystal, further supporting the assignment.

To summarize, we have added a third technique to the arsenal of recently developed in situ microspectroscopic methods for studying catalytic solids in the act. Along the lines of our recent reports ${ }^{[4,5]}$ and the present communication, a space- and time-resolved analysis of the catalytic activity within catalytic solids should be preferably undertaken as follows. In situ UV/Vis microspectroscopy offers the possi- 
bility to map the formation of reaction products in the course of a catalytic process with the potential to obtain useful optical absorption spectra allowing for a kinetic analysis of the reaction products formed. From these in situ optical absorption spectra, a three-dimensional distribution can be reconstructed using confocal fluorescent microscopy by exciting the fluorescent molecules. Finally, IR microscopy, which enables an identification of the reagents as well as reaction products, accomplishes the structural analysis, leading to mechanistic details on the chemical reaction under study. The latter could be used in the same range of temperatures as the conventional IR spectroscopy, i.e., up to several hundred degrees centigrade. ${ }^{[28]}$ As the same spectroscopic reaction cell can be used for all three microspectroscopic techniques and the conditions in which the catalytic reaction takes place remain unaltered, the results obtained with the three different techniques are fully complementary, and congruent mechanistic information can be obtained. We believe that this combined three-pronged in situ microspectroscopic approach can become an indispensable tool to study a wide variety of catalytic materials under true reaction conditions. Moreover, in situ synchrotron-based IR microspectroscopy can develop into a very powerful standalone method, owing to its universal chemical sensitivity as no light-absorbed species or fluorescent probe molecules are required for evaluating catalytic activity. To expand its application domain beyond acid-base catalytic reactions new probe reactions and related reporter molecules are under investigation in our laboratory.

\section{Experimental Section}

IR microspectroscopy. FTIR spectra and images were collected at beamline U10B at the National Synchrotron Light Source (NSLS), Brookhaven National Laboratory (Upton, NY, USA). A Thermo Nicolet Magna 860 FTIR spectrometer, coupled to a Continumm IR microscope (Thermo Nicolet, Madison, WI, USA) was used with the synchrotron light as the infrared source. The microscope was equipped with a $32 \times$ Schwarzschild objective, a motorized $x-y$ mapping stage, an adjustable rectangular aperture, and a $\mathrm{LN}_{2}$-cooled mercury cadmium telluride detector. The IR microscope stage was raster-scanned through the area with a step size of $3 \mu \mathrm{m}$ pixel. At each point, a transflectance spectrum was recorded in reflectance mode in the mid-infrared spectral range $\left(4000-800 \mathrm{~cm}^{-1}\right)$ with a spectral resolution of $8 \mathrm{~cm}^{-1}$ and 64 scans co-added. IR beam (aperture) size used for the mapping was $3 \mu \mathrm{m} \times 3 \mu \mathrm{m}$.

Materials. ZSM- 5 crystals ( $\mathrm{Si} / \mathrm{Al}$ ratio of 17$)$ were provided by Exxon-Mobil (Machelen, Belgium). Styrene derivatives (Acros Organics) were used as received. Zeolite crystals were exposed to 4-fluorostyrene and heated in an in situ cell (FTIR 600, Linkam Scientific Instruments) equipped with a temperature controller (TMS 93). Prior to reaction before the thermal treatment the crystals were scanned with the IR microscope to assure equilibrium concentration of the reagent over the crystal.

DFT calculations. Density functional theory calculations were performed using the TURBOMOLE quantum chemistry package. ${ }^{[26]}$ A geometry-optimization step preceded the calculation of the vibrational spectrum. IR frequencies were calculated within the harmonic approximation. The results are converged with respect to basis set and exchange-correlation functional (see the Supporting Information). The spectra shown in Figure 3 were calculated using the B3LYP parameterization of the exchange-correlation functional and a TZVP basis set, and the calculated vibrational frequencies were scaled by a factor of $0.97 .^{[27]}$

Received: December 5, 2007

Published online: March 28, 2008

Keywords: heterogeneous catalysis - IR microscopy ·

oligomerization $\cdot$ synchrotron radiation $\cdot$ zeolites

[1] a) M. T. Reetz, M. H. Becker, K. M. Kuhling, A. Holzwarth, Angew. Chem. 1998, 110, 2792-2795; Angew. Chem. Int. Ed. 1998, 37, 2647-2650; b) C. Lamberti, C. Prestipino, F. Bonino, L. Capello, S. Bordiga, G. Spoto, A. Zecchina, S. D. Moreno, B. Cremaschi, M. Garilli, A. Marsella, D. Carmello, S. Vidotto, G. Leofanti, Angew. Chem. 2002, 114, 2447-2450; Angew. Chem. Int. Ed. 2002, 41, 2341-2344; c) A. Suzuki, Y. Inada, A. Yamaguchi, T. Chihara, M. Yuasa, M. Nomura, Y. Iwasawa, Angew. Chem. 2003, 115, 4943-4947; Angew. Chem. Int. Ed. 2003, 42, 4795-4799; and M. Tada, S. Murata, T. Asakoka, K. Hiroshima, K. Okumura, H. Tanida, T. Uruga, H. Nakanishi, S. Matsumoto, Y. Inada, M. Nomura, Y. Iwasawa, Angew. Chem. 2007, 119, 4388-4393; Angew. Chem. Int. Ed. 2007, 46, 43104315 .

[2] a) M. B. J. Roeffaers, B. F. Sels, H. Uji-i, F. C. De Schryver, P. A. Jacobs, D. E. De Vos, J. Hofkens, Nature 2006, 439, 572-575; b) B. M. Weckhuysen, Nature 2006, 439, 548-548.

[3] M. B. J. Roeffaers, B. F. Sels, H. Uji-i, B. Blanpain, P. L'hoëst, P. A. Jacobs, F. C. De Schryver, J. Hofkens, D. E. De Vos, Angew. Chem. 2007, 119, 1736-1739; Angew. Chem. Int. Ed. 2007, 46, $1706-1709$.

[4] M. H. F. Kox, E. Stavitski, B. M. Weckhuysen, Angew. Chem. 2007, 119, 3726-3729; Angew. Chem. Int. Ed. 2007, 46, $3652-$ 3655 .

[5] E. Stavitski, M. H. F. Kox, B. M. Weckhuysen, Chem. Eur. J. 2007, 13, 7057-7065.

[6] L. Karwacki, E. Stavitski, M. H. F. Kox, J. Kornatowski, B. M. Weckhuysen, Angew. Chem. 2007, 119, 7366-7369; Angew. Chem. Int. Ed. 2007, 46, 7228-7231.

[7] M. B. J. Roeffaers, G. De Cremer, H. Uji-i, B. Muls, B. F. Sels, P. A. Jacobs, F. C. De Schryver, D. E. De Vos, J. Hofkens, Proc. Natl. Acad. Sci. USA 2007, 104, 12603-12609.

[8] M. H. F. Kox, E. Stavitski, J. C. Groen, J. Pérez-Ramírez, F. Kapteijn, B. M. Weckhuysen, Chem. Eur. J. 2008, 14, 1718-1725.

[9] J. C. Jansen, R. de Ruiter, E. Biron, H. Van Bekkum in Zeolites; Facts, Figures, Future (Eds.: P. A. Jacobs, R. A. van Santen), Elsevier, Amsterdam, 1989.

[10] M. Nowotny, J. A. Lercher, H. Kessler, Zeolites 1991, 11, 454 459.

[11] F. Schüth, J. Phys. Chem. 1992, 96, 7493-7496.

[12] G. Muller, J. Bodis, J. Kornatowski, Microporous Mesoporous Mater. 2004, 69, $1-7$

[13] P. Kortunov, C. Chmelik, J. Karger, R. A. Rakoczy, D. M. Ruthven, Y. Traa, S. Vasenkov, J. Weitkamp, Adsorption 2005, 11, 235-244.

[14] L. Heinke, C. Chmelik, P. Kortunov, D. M. Ruthven, D. B. Shah, S. Vasenkov, J. Karger, Chem. Eng. Technol. 2007, 30, 995-1002.

[15] W. D. Duncan, G. P. Williams, Appl. Opt. 1983, 22, 2914-2923.

[16] Diffraction limit in the mid-infrared region is estimated to be 2$10 \mu \mathrm{m}$ (see L. M. Miller, R. J. Smith, Vib. Spectrosc. 2005, 38, $237-240$ ). For the relevant spectral region (around $1500 \mathrm{~cm}^{-1}$ ) the best attainable spatial resolution is $3-4 \mu \mathrm{m}$.

[17] L. Fernández, V. Marti, H. Garcia, Phys. Chem. Chem. Phys. 1999, 1, 3689-3695 (Figure 6).

[18] K. B. Yoon, J. L. Lim, J. K. Kochi, J. Mol. Catal. 1989, 52, 375 386. 
[19] S. S. Pollack, R. F. Sprecher, E. A. Frommell, J. Mol. Catal. 1991, 66, $195-203$.

[20] F. L. Cozens, R. Bogdanova, M. Regimbald, H. Garcia, V. Marti, J. C. Scaiano, J. Phys. Chem. B 1997, 101, 6921-6928.

[21] V. J. Rao, N. Prevost, V. Ramamurthy, M. Kojima, L. J. Johnston, Chem. Commun. 1997, 2209-2210.

[22] V. Fornes, H. Garcia, V. Marti, L. Fernandez, Tetrahedron 1998, $54,3827-3832$.

[23] G. D. Price, J. J. Pluth, J. V. Smith, J. M. Bennett, R. L. Patton, J. Am. Chem. Soc. 1982, 104, 5971-5977.

[24] D. G. Hay, H. Jaeger, K. G. Wilshier, Zeolites 1990, 10, 571-576.
[25] C. Weidenthaler, R. X. Fischer, R. D. Shannon, O. Medenbach, J. Phys. Chem. 1994, 98, 12687-12694.

[26] R. Ahlrichs, M. Bär, M. Häser, H. Horn, C. Kölmel, Chem. Phys. Lett. 1989, 162, 165.

[27] A. P. Scott, L. Radom, J. Phys. Chem. 1996, 100, 16502-16513.

[28] Examples of high-temperature IR microspectroscopy up to $900 \mathrm{~K}$ can be found in H. Yamagishi, S. Nakashima, Phys. Chem. Miner. 1997, 24, 66-74. At higher temperatures black-body radiation is not negligible. However, it could be compensated by recording a background reference spectrum at the same temperature. 